\title{
Zwischen individuellen Ansprüchen und systemischen Erfordernissen: der Konsum von tierlichen Produkten in der Ernährung
}

Michael Rosenberger

Die Frage der Tiernutzung und der Tiertötung kann man mit Fug und Recht als die „Gretchenfrage“ der Tierethik bezeichnen. An ihr entzünden sich hochemotionale Debatten, an ihr scheiden sich die Geister. Auf sie laufen letztlich alle bisher behandelten Themen dieses Kongresses zu, so dass häufig nur nach dem diesbezüglichen status confessionis einer Person oder Gruppe gefragt wird. Die Argumente, die zu einer Position führen, fallen dabei leicht unter den Tisch, ja sie werden mitunter nur als Scheinargumente verstanden, die nachträglich eine bereits feststehende Position rechtfertigen sollen.

All diesen Dynamiken zum Trotz dürfen wir uns vor der Frage nicht drücken. In einem biozentrischen Ansatz, wie ich ihn vertrete, geht es dabei nicht um die menschliche Lust auf Fleisch oder Milch(-produkte). Überhaupt geht es in einem solchen Ansatz nicht primär um individuelle Interessen einzelner Menschen an der Tiernutzung - die könnten die Grundbedürfnisse der Tiere nicht so weit aushebeln, dass man sie nutzen oder gar töten könnte. Die entscheidenden Argumente zu Gunsten einer Tiernutzung sind - zumindest jenseits einer materialen Anthropozentrik ökologischer Natur und damit systemische, überindividuelle Argumente. Das Knifflige an den Fragen der Nutzung oder des Nutzungsverzichts von Tieren für die menschliche Ernährung ist in dieser Perspektive, dass sie im Schnittfeld individueller und systemischer Erfordernisse und Konsequenzen liegen. Eine Betrachtung nur unter einer der beiden Dimensionen kann ihrer Komplexität nicht gerecht werden.

Mein Beitrag gliedert sich daher in zwei Teile: Zunächst referiere ich ökosystemische Argumente für die Legitimität der Tiernutzung/-tötung zu Nahrungszwecken unter bestimmten Bedingungen (1) - hier greife ich weitgehend auf bereits publizierte Thesen zurück (vgl. Michael Rosenberger 2015, 183-188 und 2016, 94-98). Eine vertiefte Aufklärung meines Argumentationsgangs ergibt sich im zweiten Schritt durch Betrachtung aus der Metaperspektive gerechtigkeitstheoretischer Analysen (2) - hier präsentiere ich Gedanken, die ich zumindest in dieser Deutlichkeit noch nicht 
publiziert habe. Nicht behandeln kann ich aus Zeitgründen die im Hintergrund relevante Verhältnisbestimmung von Individualwohl und Gemeinwohl, wie sie im klassischen Gemeinwohlprinzip der christlichen Sozialethik ausgedrückt ist. Dies habe ich andernorts bereits getan (Michael Rosenberger 2018) und will hier nur darauf verweisen.

\section{1. Ökosystemische Argumente für die Tiernutzung}

Ethische Überlegungen setzen an bei realen, vorhandenen Konflikten und Konkurrenzsituationen um knappe Güter. John Rawls zählt diese zu den „Anwendungsverhältnissen der Gerechtigkeit“ (John Rawls 1975, 148-152, $\$ 22)$. Und sie bestimmen auch die Frage nach der Tiernutzung und Tiertötung. Denn wie alle Tiere lebt der Mensch - ob Vegetarier, Veganer oder nicht - von der Tötung anderer Lebewesen. Will er leben, ist er gezwungen, Gewalt gegen andere Lebewesen anzuwenden. „An einem späteren, aber durchaus bestimmten Punkt ist auch der Vegetarier rücksichtslos gegen das Leben, das ihn nähren soll. [...] Nur tot kann das andere Wesen den Menschen ernähren“ (Gottfried Bachl 2008, 35). Oder etwas salopper formuliert:

„Die Idee, dass es eine Ernährungsweise gibt, die niemandem schadet, ja, die den Tod völlig ausklammert und nur immer wieder neues Leben schafft, ist eine Illusion. Dies gilt selbst für eine vegane Ernährungsweise. [...] Selbst eine fruktarische Diät [...] ist keine Ausnahme. [...] Die Nahrungskette ist nicht politisch korrekt. Sie folgt einer ebenso einfachen wie genialen Logik: Tod und Leben sind keine Widersprüche, sie ergänzen einander“" (Theresa Bäuerlein 2011, 29-30).

Der Mensch kann versuchen, mit einem Minimum an Gewalt gegenüber den Lebewesen auszukommen und diese ehrlich zu rechtfertigen. Er kann ihr aber nicht gänzlich ausweichen. Das spricht prima facie im Sinne der Übelminimierung für den Vegetarismus, aber nicht prinzipiell, sondern nur tendenziell. Zwischen vegetarischer und nichtvegetarischer Ernährung lässt sich - selbst wenn man die zahlreichen direkt durch Ackerbau (Bodenbearbeitung, Spritz- und Düngemittel, Erntemaschinen) getöteten Tiere einmal außen vor lässt - nur ein relativer bzw. gradueller Unterschied machen. Denn auch Pflanzen sind höchst komplexe Lebewesen, deren Kommunikation und „Intelligenz“ erst in den letzten Jahren zu erforschen 
begonnen wird. ${ }^{1}$ Schon die ersten Ergebnisse sind faszinierend und stellen den tiefen Graben zwischen Pflanzen und Tieren in Frage, den wir in den letzten Jahrhunderten ziehen. Als Vertreter einer biozentrischen Position ist für mich klar, dass Pflanzen ebenso Mitgeschöpfe des Menschen sind wie Tiere und deshalb in eine ethische Gesamtbetrachtung einbezogen werden müssen.

Der springende Punkt einer ethischen Gesamtbetrachtung ist aber (immer!), dass die Betrachtung individueller Beziehungen zwischen Mensch und Tier ergänzt wird durch die Betrachtung der systemischen Wechselwirkungen. Handelnde und behandelte Individuen sind Teil eines größeren Ganzen. Die systemische Betrachtungsweise sieht Mensch und Tier als Teil der ökologischen Kreisläufe und Wechselbeziehungen. Folgende Gesichtspunkte stechen heraus:

1) Der ökologische Landbau, der zweifelsohne die einzige nachhaltige und damit auch den (Wild-)Tieren dienliche Weise der Landwirtschaft darstellt, kann keinen Acker ohne den natürlichen Dünger aus dem Mist seines Viehs bewirtschaften. Bisher gibt es kein erprobtes Konzept des ökologischen Landbaus, das ohne Tiernutzung auskommt. Denn eine Kreislaufwirtschaft braucht das Wechselspiel von Boden und Tieren: Die Tiere ernähren sich von dem, was auf den Böden des Ökohofs wächst, und ihr Mist dient als Düngemittel, das dem Boden die wesentlichen Stoffe wieder zurückgibt.

2) Global betrachtet sind viele landwirtschaftlich nutzbaren Flächen nicht zum Lebensmittelanbau für den Menschen, wohl aber zum Futtermittelanbau für das Vieh geeignet - man denke an Almen und Flächen in Steillage, an Feuchtwiesen und andere nicht beackerbare Böden. Einschlägigen Rechnungen zufolge sind 69 Prozent aller weltweit landwirtschaftlich nutzbaren Flächen Weideflächen, 28 Prozent Ackerflächen und drei Prozent Dauerkulturen. Würde man die Weideflächen unbewirtschaftet lassen, wäre ein erheblicher Verlust an Nahrungsmitteln für den menschlichen Verzehr die Folge. Tiere müssen nicht mit Getreide und anderen Futtermitteln vom Acker gefüttert werden, die die Menge menschlicher Lebensmittel reduzieren - im Gegenteil: „Grass-fed beef“" ist gesünder und schmeckt besser. Würden die Weiden aber nicht genutzt, würde sich

1 Einen guten Überblick dazu und zu den ethischen Konsequenzen für die Interaktion zwischen Mensch und Pflanze findet sich auf der Homepage des Forschungsprojekts „New Directions in Plant Ethics“ der Universität Wien (Prof.in Angela Kallhoff): https://plantethics.univie.ac.at/ (abgerufen am 15.10.2019). 
zwangsläufig der Nutzungsdruck auf bisher nicht genutzte Naturgebiete erhöhen - zum Schaden der dort lebenden Wildtiere.

3) Die Nichtbewirtschaftung von Weiden würde auch einen enormen Verlust an Biodiversität verursachen. Denn nachhaltig genutzte Weideflächen und Mähwiesen tragen in höchstem Maße zur Vielfalt von Arten und Biotopen bei. Für Europa hat man in der Fauna-Flora-Habitat-Richtlinie 92/43/EWG im Jahr 1992 etwa 200 verschiedene erhaltenswerte Lebensraumtypen aufgelistet. Davon sind etwa 30 Prozent nur durch nachhaltige Tierhaltung erhaltbar, die zusammengerechnet die Hälfte aller Flächen ausmachen (Rainer Luick 2014, 147). Auf ihnen leben ungezählte Tiere, die sonst keinen Lebensraum hätten. - Wohlgemerkt geht es in all meinen Überlegungen zur Beweidung um (aufs Ganze gesehen) nachhaltige Formen. Die Düngung von Wiesen und Weiden mit Gülle und der nachfolgend häufigere Schnitt sind Intensivierungsmaßnahmen, die zur Verarmung der Biodiversität führen. Sie gilt es mittelfristig zu eliminieren.

4) Eine ganzjährige nachhaltige Weidehaltung von Milchkühen ist zugleich klimaverträglicher als die Stallhaltung. Das ergab ein Forschungsprojekt des amerikanischen Landwirtschaftsministeriums an der Universität Pennsylvania (United States Department of Agriculture [USDA] 2011). Obwohl die Kühe auf der Weide nur 6000 Kilogramm Milch pro Jahr erzeugen, während es ihre Artgenossinnen in der Stallhaltung auf $10.000 \mathrm{Ki}$ logramm bringen, sind die Emissionen der Treibhausgase Methan, von Stickoxiden und Kohlendioxid pro Kilogramm Milch in ganzjähriger Weidehaltung um acht Prozent niedriger als in intensiver Stallhaltung. Die Ammoniakemissionen pro Kilogramm Milch fallen sogar um 30 Prozent geringer aus, der Kohlendioxid-Ausstoß um sechs Prozent.

5) Schließlich erhöht die nachhaltige Weidehaltung auch die Bodenfruchtbarkeit - ein Schlüsselfaktor zukunftsfähiger Landwirtschaft. Während die Überweidung von Böden zur Bodenerosion führt, hat die Unterweidung kurzfristig die Verbuschung und langfristig die Bewaldung zur Folge. Doch „die weltweit fruchtbarsten Böden [...] sind ehemalige Steppenböden" (Anita Idel 2014, 154), also Böden, die lange Zeit nachhaltig beweidet wurden. Und daraus folgt in ökosystemischer Perspektive: „Gras braucht die Graser" (Anita Idel 2014, 153). - Natürlich könnte man (gegen die vegane, aber im Sinne der vegetarischen Option) versuchen, diese Flächen ausschließlich für Milchvieh zu nutzen. Dann könnte man aber die männlichen Tiere, die es ja ebenfalls gibt, nicht mehr brauchen. Und da ist es womöglich doch besser, mittels Zweinutzungsrassen beide Geschlechter zu nutzen und nicht die eine Hälfte der Nachkommen gleich zu schlachten. 
6) Eine weitere ökosystemisch wichtige Komponente ist die Jagd. Jagdbare Tiere haben heute in den Industrieländern kaum noch natürliche Fressfeinde und vermehren sich selbst ohne menschliche Fütterung oft so stark, dass sie sogar in einer sehr naturnahen Forstwirtschaft die Baumbestände gefährden. Jagd reguliert die Tierbestände - wenn sie sachgerecht und waidgerecht ausgeübt wird. Das dient jenen Tieren, die nicht geschossen werden, denn sie finden dann ein besseres Nahrungsangebot. Natürlich, in der realen Praxis des Jagens liegt manches im Argen. Das ist aber kein prinzipielles Argument gegen die Jagd, sondern eher eines für eine reflektierte Jagdethik (Michael Rosenberger 2008 und 2019).

7) Schließlich ist auch an die Fischerei zu denken: Über 70 Prozent der Erdoberfläche sind Wasser, der größte Teil davon Meere. Sie speichern einen Großteil der Sonnenenergie, die auf die Erde trifft. Diese Speicherung erfolgt aber (neben der thermischen Speicherung) vor allem in Tieren, nicht so sehr in Pflanzen wie auf der Landfläche. Zwar sind derzeit etwa ein Drittel der 450 befischten Arten in den Weltmeeren überfischt und nur sieben Prozent „unterfischt“ (Food and Agriculture Organization 2018) - aber können wir ohne Fischfang auskommen, wenn wir die Menschheit ernähren wollen? Ganz nebenbei profitieren von nachhaltigem Fischfang auch viele Meerestiere, mitunter sogar die Fische der befischten Art selbst.

Beide Betrachtungsweisen, die tierethisch-individuelle wie die ökonomisch-ökologisch-systemische, verhindern in ihrer Verbindung wenigstens nach heutigem Kenntnis- und Möglichkeitsstand eine generelle Ablehnung der Tiernutzung und Tiertötung. Die konkrete Art und Weise unserer Tierwirtschaft stellen sie aber gewaltig in Frage. Denn der Großteil der Viehwirtschaft ist keine nachhaltige Weidewirtschaft, der Großteil der Landwirtschaft kein Ökolandbau und der Großteil der Fischerei weit weg von nachhaltiger Nutzung.

Was ergibt sich daraus an Forderungen für den Konsum tierlicher Produkte zu Nahrungszwecken (Michael Rosenberger 2015, 197-206)? Wollen wir die gesamte Nutztierhaltung als nachhaltige Weidehaltung gestalten sowie Jagd und Fischfang ebenfalls nachhaltig betreiben, bedarf es nach übereinstimmender Schätzung der meisten Fachleute in den Industrieländern ungefähr einer Viertelung des Fleischverzehrs: 15 Kilogramm netto bzw. 20 Kilogramm brutto („Fleischverbrauch“) statt 60 bzw. 80 Kilogramm pro Kopf und Jahr. Analog müsste auch der Konsum von Milchprodukten und Eiern deutlich zurückgefahren werden. Biozentrisch-ökologisch argumentierende TierschützerInnen und überzeugte VeganerInnen können also noch lange einen gemeinsamen Weg gehen, ehe die Debatte eines kompletten Verzichts auf die Tiernutzung politisch relevant wird. 
Im Kontext einer theologischen Ethik können drei Elemente der alten spirituellen Tradition der Kirche den Weg zur Viertelung des Konsums tierlicher Produkte unterstützen:

1) Die Tradition fleischfreier Wochentage und Zeiten als rituelles Zeichen des Bemühens um Mäßigung (Michael Rosenberger 2016, 128-132). Es scheint mir paradox, dass die Initiative für einen fleischfreien Wochentag in den meisten Ländern von der Umweltbewegung, nicht von den Kirchen ausging. Erst recht ist die Bedeutung des Begriffs „Karneval“ - „Fleisch, lebe wohl“ - in Vergessenheit geraten. Dabei ging es die längste Zeit der Kirchengeschichte vierzig Tage ohne Fleisch, wie überhaupt noch bis ins 19. Jahrhundert etwa die Hälfte aller Tage des Kirchenjahres gebotene Abstinenztage waren.

2) Eine Tradition des frühen Mönchtums war es, die vegetarische/vegane Lebensweise eng mit Ehelosigkeit und Armut verbunden zu sehen. Auf heute übertragen verdient sie daher den Status eines evangelischen Rates (Michael Rosenberger 2016, 98-101 und 136-141): Denen ans Herz gelegt, denen das Charisma dafür geschenkt ist, mit dem Anspruch auf Hochschätzung durch alle anderen, weil darin ein Wesenszug des Evangeliums und ein Vorgeschmack auf den Himmel deutlich wird.

3) Die Eucharistie - das zentrale Sakrament des Christentums - ist eine vegane Speise. Dabei waren ihre Beschränkung auf Brot und Wein und der Verzicht auf tierliche Lebensmittel keineswegs zwingend, sondern kristallisierten sich nach einem längeren Prozess des Suchens und Ausprobierens heraus. Sobald die Eucharistie aber in eine "fleischlastige" Kultur implementiert wird, gewinnt sie den Charakter eines Gegensymbols. Sie hinterfragt, ob Fleisch wirklich unverzichtbarer Bestandteil eines guten Essens sein muss.

Was sich aus ökologisch-biozentrischer Perspektive als absolut geboten erweist, kann also nahtlos an die normativen Traditionen des Christentums für den Konsum tierlicher Produkte anknüpfen. Die Begründung ist teilweise eine andere, aber das Ergebnis ist dasselbe.

\section{Die Betrachtung aus der Metaperspektive von John Rawls}

Nun argumentiere ich wie z. B. auch Mark Rowlands in meiner Tierethik stark mit der Gerechtigkeitstheorie von John Rawls. Die These von Martha Nussbaum, Rawls' Gerechtigkeitstheorie eigne sich prinzipiell nicht zur Begründung einer Tierethik (Martha Nussbaum 2010), teile ich nicht und ebenso wenig die Kritik von Daniel Loewe 2008 an Mark Rowlands (vgl. Michael Rosenberger 2015, 135-143). Vielmehr nehme ich Rawls' eigene 
Aussage ernst, dass seine Theorie der Gerechtigkeit prinzipiell auf Tiere erweiterbar sei (John Rawls 1975, 556).

\subsection{Die Theorie der Gerechtigkeit als Institutionenethik}

In einer bestimmten Weise scheint mir der Rawls'sche Ansatz für die Entwicklung einer Tierethik sogar besser geeignet als die meisten anderen Normtheorien. Denn die Tierethik steht wie gezeigt im Spannungsfeld individueller und systemischer Erfordernisse. Und genau auf dieses Spannungsfeld richtet eine Institutionenethik wie die von John Rawls besondere Aufmerksamkeit - anders als die Diskursethik von Jürgen Habermas, dessen „gattungsethische“ Überlegungen der jüngeren Zeit als nachträglicher Reparaturversuch genau dieser Schwachstelle gelesen werden können. Rawls' Institutionenethik darf nicht dem moralischen Individualismus zugerechnet werden (vgl. dazu den Beitrag von Herwig Grimm in diesem Band). ${ }^{2}$

Es war Rawls' erstes Aha-Erlebnis auf dem Weg seiner Theoriebildung, dass an die Stelle einer isolierten Betrachtung einzelner Handlungen oder Normen die holistische Betrachtung von Regeln innerhalb der Regelsysteme, in die sie eingebettet sind, treten muss. So formuliert er es bereits 1955 in seinem berühmten, noch utilitaristisch grundierten Aufsatz „Two Concepts of Rules“. In der Theorie der Gerechtigkeit wird daraus zwei Jahrzehnte später die grundlegende Option, das Materialobjekt auf gerechte Institutionen zu fokussieren (John Rawls 1975, 23-27, \$2). Institutionen sind Regelsysteme. Die Parteien im Urzustand können keine einzelnen Regeln wählen, sondern nur Pakete in sich konsistenter Regelsysteme, eben Institutionen. Das verhindert das Rosinenpicken, was für die tierethische Konkretion einschneidende Folgen hat. ${ }^{3}$

2 Klassische Normtheorien kranken meines Erachtens häufig an einer Unterbelichtung der systemischen Aspekte für die Normbegründung. Normen stehen untereinander in Zusammenhang - eine isolierte Betrachtung und Begründung einer Einzelnorm wird diesem Zusammenhang nicht gerecht.

3 Martin M. Lintner 2017, 125 behauptet von der Rawls'schen Gerechtigkeitstheorie: „In Bezug auf die Tierethik jedoch würde dieser Ansatz, konsequent weitergedacht, zu einem absoluten Verbot der Tötung von Tieren führen. Auf der individualethischen Ebene ist nämlich davon auszugehen, dass kein Lebewesen im fiktiven Urzustand unter dem ,Schleier des Nichtwissens' [...] der eigenen Tötung zustimmen würde.“ Wie die folgenden Überlegungen zeigen sollen, wäre Lintners These richtig, wenn es Rawls um gerechte Einzelnormen und nicht um gerechte Institutionen ginge. 


\subsection{Gerechtigkeit heißt nicht Vollkommenheit}

Eine zweite Grundeinsicht der Gerechtigkeitstheorie ist die, dass es in Normtheorien um Gerechtigkeit geht und nicht um Perfektion. Rawls hat dieser Frage einen eigenen Paragrafen seines Werks gewidmet (John Rawls 1975, 360-367, \50). Er reflektiert darin, ohne das ausdrücklich zu sagen, den Unterschied zwischen Normethik und Tugendethik. Tugendethik

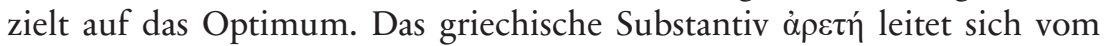

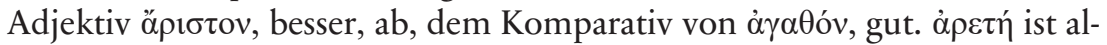
so diejenige Eigenschaft, durch die etwas „besser“ oder „bestens“, „optimal“ ist. Normen der Gerechtigkeit hingegen geben sich mit weniger zufrieden, so Rawls, weil sie zwischen unterschiedlichen Vorstellungen des Optimums vermitteln müssen.

\subsection{Gerechtigkeit und individuelle „Grundfreiheiten“}

Nun kommt in der Rawls'schen Gerechtigkeitstheorie allen Vertragsparteien eine Liste von Grundfreiheiten zu, die so umfangreich ist, wie das mit dem gleichen System der Grundfreiheiten für alle anderen kompatibel ist (John Rawls 1975, 81-86). Diese Grundfreiheiten sind in der lexikalischen Ordnung der Gerechtigkeitsgrundsätze den Fragen wirtschaftlicher Ungleichheit vorgeordnet und können nicht gegen diese abgewogen werden (John Rawls 1975, 62-63 und 81-86). Insofern ist die Frage, die Kurt Remele aufwirft ${ }^{4}$, durchaus von zentraler Bedeutung: Müssen nicht die Tiere Grundrechte besitzen, die wie die Menschenrechte konkreten Güterabwägungen vorgeordnet sind, also nur gegen die Grundrechte anderer Tiere einschließlich des Menschen abgewogen werden dürfen? Dabei dürfte es ziemlich gleich sein, ob man hier juristische oder moralische Rechte meint. Solange man nicht rechtspositivistisch argumentiert, müsste eine hohe Konkordanz der Argumentation bestehen.

Folgende Überlegungen scheinen mir zur Beantwortung dieser Frage relevant:

1) Menschenrechte sind Rechtsansprüche von Menschen gegenüber Menschen - sie funktionieren zunächst einmal innerhalb einer einzelnen Spezies: Für „Menschenrechte ist zunächst nur definitorisch, dass sie strikt allen Menschen zukommen. Ob es Gründe gibt, gleichartige Rechte auch Tieren

4 Vgl. den Beitrag von Kurt Remele im vorliegenden Band. 
zuzusprechen, ist eine andere Frage“ (Andreas Wildt 1998, 136; vgl. Saskia Stucki 2016, 342).

2) Eine Speziesethik (bei Habermas biologisch unpräzise „Gattungsethik“ genannt) ist noch lange kein Speziesismus. Dieser bedeutet per definitionem die Abwertung anderer Spezies (wie Rassismus die Abwertung anderer Rassen und Sexismus die Abwertung des anderen Geschlechts). Eine Speziesethik, die zwischen Menschenrechten und Rechten anderer Tiere unterscheidet, wertet die nichtmenschlichen Spezies keineswegs automatisch ab. Denn Menschenrechte werden nicht mit einem höheren Wert der Menschen begründet, ja überhaupt nicht mit Werten, sondern bestenfalls mit der Würde. Nach Immanuel Kant ist die Würde aber gerade das, was keinen Preis hat, also nicht verrechenbar und nicht vergleichbar ist. Sie erschließt sich nicht aus einem Werturteil und erst recht nicht aus bestimmten Eigenschaften, die zwangsläufig nicht alle Menschen hätten. Vielmehr begründen sich Menschenwürde und Menschenrechte aus Erwägungen zur Stabilität und Symmetrie eines Beziehungsgefüges innerhalb der Spezies Mensch, wie dies die "Gattungsethik“ von Habermas deutlich macht (Jürgen Habermas 2001). Eine Speziesethik, die die Menschenrechte von Grundrechten für Tiere unterscheidet, wertet also die Tiere nicht ab, sondern erkennt nur an, dass es innerartlich andere und höhere Erfordernisse für die Stabilität von Beziehungsgefügen gibt als artübergreifend.

3) „Mensch“ ist eine Speziesbezeichnung, „Tier“ nicht. Von Menschenrechten auf Grundrechte für Tiere zu schließen, ist daher ein äußerst komplexer Vorgang, der zusätzliche Annahmen braucht. Denn Tierrechte sollen Rechte der Tiere aller Spezies einzig und allein gegenüber dem Menschen sein, also nur einer Spezies gegenüber und noch dazu einer für die meisten Tiere fremden. Das macht die Forderung von Grundrechten für Tiere nicht unmöglich - sie braucht aber einen viel längeren Anlauf als den Verweis auf Menschenrechte. Eine sehr sorgfältige und für mich einleuchtende Argumentation liefert Saskia Stucki (2016, 333-394; vgl. auch Anne Peters 2019, 129-132). Ich selber habe mich in vorangehenden Publikationen in Rawls'scher Terminologie für „Grundfreiheiten“ für Tiere ausgesprochen (Michael Rosenberger 2015, 141-142) - das meint genau das, was Stucki und Peters „Grundrechte“ für Tiere nennen. Werden solche anerkannt, ergeben sich viel strengere Rechtfertigungspflichten für den menschlichen Umgang mit Tieren als im geltenden Tierschutzrecht (Saskia Stucki 2016, 358; Anne Peters 2019, 130).

4) Welchen rechtssystematischen Stellenwert haben aber solche Grundrechte der Tiere? An Kurt Remeles Überlegung ist zutreffend, dass Menschenrechte (und analog dann auch Grundrechte der Tiere) nicht gegen untergeordnete Individual- oder Kollektivrechte wie z. B. wirtschaftliche 
Vorteile abgewogen werden dürfen. Wohl aber werden sie gegeneinander abgewogen, denn sie stehen nicht selten miteinander im Konflikt bzw. zueinander in Konkurrenz. Zudem - und das ist noch bedeutsamer - werden Menschenrechte auch gegen übergeordnete Aspekte des Gemeinwohls abgewogen. Dabei meint Gemeinwohl nicht die Summe des Einzelwohls der Individuen, sondern dessen strukturelle, systemische Möglichkeitsbedingung (vgl. Karlheinz Ruhstorfer 2012, 263; Valentin Zsifkovits 2012, 83). „Grundrechte weisen zwar eine erhöhte Eingriffsresistenz auf, gewähren allerdings [...] keinen absoluten Schutz, sondern können im Interesse der Allgemeinheit oder zum Schutz von Grundrechten Dritter unter Einhaltung bestimmter Voraussetzungen eingeschränkt werden" (Saskia Stucki 2016, 356).

5) Der Erhalt des Ökosystems ist genau eine solche Möglichkeitsbedingung des Einzelwohls: Gerät das Ökosystem aus der Balance, werden viele in ihm lebende Wesen zugrunde gehen. Folgerichtig nennt auch Saskia Stucki „Umwelt- und Artenschutz“ als legitime Begründung für eine Einschränkung menschlicher wie tierlicher Grundrechte zu Gunsten des öffentlichen Interesses (Saskia Stucki 2016, 358). Ethisch wie juristisch halte ich es für geboten (!), für den Erhalt des Lebenshauses der Erde manche Grundrechte der Tiere (und auch manche der Menschen!) zu overrulen. Konkrete Beispiele habe ich im ersten Unterkapitel angeführt.

\subsection{Die Theorie der Gerechtigkeit als Institutionenvergleich}

Nach Rawls werden sich die Parteien im Urzustand in verschiedene Institutionen desselben Typs - und das wären in unserem Fall Tierhaltungssysteme - mit all ihrer Kreativität und Phantasie fiktional hineinversetzen. Sie werden die Institutionen vergleichen und schließlich eine unter mehreren gerechten wählen. ${ }^{5}$ Dabei müssen sie nicht alle denkbaren Institutionen in eine lineare Reihung bringen. Wohl aber müssen sie durch ihren Vergleich beurteilen, ob sie diese im Großen und Ganzen für gerecht oder für ungerecht halten, also für wählbar oder nicht wählbar. Hauptkriterium ist

5 Mark Rowlands ${ }^{2}$ 2009, 174 führt dasselbe Gedankenexperiment durch. Allerdings bietet er nur zwei Alternativen an: Eine Welt mit und eine Welt ohne industrielle Massentierhaltung. In diesem Fall, so seine These, werden die Individuen im Rawls'schen Urzustand die Welt ohne industrielle Massentierhaltung wählen. Bis hierhin stimme ich Rowlands zu. Dann allerdings schließt er daraus, dass Tierhaltung und Tiertötung generell ethisch abzulehnen seien. Dieser Schluss ist voreilig: Tertium datur! 
dabei nach Rawls das maximum minimorum (John Rawls 1975, 174-185, $\$ 26$ ), d. h. eine relativ günstige Situation der im jeweiligen Haltungssystem am schlechtesten gestellten Tiere. Wie also könnte ein solcher Institutionenvergleich sehr vereinfacht aussehen?

1) Die Trennlinie zwischen ungerechten und gerechten Tierhaltungssystemen, also gleichsam der "Nullpunkt“, ist die Nichtexistenz des betreffenden Tieres: Wenn ein Haltungssystem so beschaffen ist, dass die Parteien im Urzustand die Nichtexistenz vernünftigerweise dem Leben als Tier in diesem System vorziehen würden, muss das System als (mehr oder weniger, aber eben doch) ungerecht bezeichnet werden. Wenn ein Haltungssystem so beschaffen ist, dass die Parteien im Urzustand ein Leben als Tier in diesem System vernünftigerweise der Nichtexistenz vorziehen würden, kann das System als (mehr oder weniger, aber eben doch) gerecht bezeichnet werden.

2) Ein erstes, nämlich das ursprüngliche „Haltungssystem“ aller Tiere ist das Leben in freier, vom Menschen nicht beeinflusster Wildbahn. In den meisten Weltregionen ist diese heutzutage verschwunden, weil der Mensch die Ökosysteme beeinflusst - wir reden vom „Anthropozän“ -, aber es dient als eine heuristisch fruchtbare und grundsätzlich vorstellbare Fiktion. Für die theologische Ethik ist diese „Tierhaltungsform Gottes“ eindeutig unter die lebenswerten, „gerechten“ Systeme einzuordnen, da wir an einen gerechten Schöpfer glauben (wohl wissend, dass göttliche und menschliche Gerechtigkeit nicht univok verstanden werden dürfen). Dabei müssen wir nicht wie Gottfried Wilhelm Leibniz annehmen, dass es sich um die beste aller möglichen Welten handelt. Es genügt, dass sie „gerecht“ ist, d. h. (bei aller Offenheit der Theodizeefrage!) für alle Individuen im Großen und Ganzen lebenswert. Diese These wird allerdings schnell auf eine harte Probe gestellt, wenn wir das Tierhaltungssystem Gottes an den am schlechtesten gestellten Individuen messen: $\mathrm{Da}$ ist das Adlerjunge, das von seinem Bruder oder seiner Schwester solange gehackt und malträtiert und bei der Fütterung durch die Eltern abgedrängt wird, bis es elend verhungert; die junge Gämse, die bereits in ihren ersten Lebenstagen von einem Fressfeind gejagt und verspeist wird; der alte Wolf, der in einem wochenlangen Ringen immer schwächer wird und einsam verendet. Wohlgemerkt: Auch das stärkere, überlebende Adlerjunge, der Fressfeind der Gämse und das Rudel des Wolfes haben es schwer und sichern gerade mal so ihr eigenes Überleben - man kann hier keineswegs von ausbeuterischem Verhalten reden. Aber wir sollten uns doch von naiver Harmonie verabschieden, wenn wir über die freie Wildbahn reden. Diese Überlegung verschärft sich noch, wenn wir berücksichtigen, dass etwa die Hälfte der erstjährigen Gämsen im Winter umkommt - durch Lawinen, nicht durch Fressfeinde. Die Rede 
von der Gutheit der Schöpfung, biblisch in Gen 1 grundgelegt, ist eine extrem steile Glaubensaussage.

3) Die Massen- oder Intensivtierhaltung hingegen müssen wir eindeutig auf der Seite ungerechter Tierhaltungssysteme verorten. $\mathrm{Zu}$ viele, um nicht zu sagen: fast alle Güter werden den Tieren genommen, damit der Nutzen für den Menschen maximiert wird. Allen guten Absichten der einschlägigen EU-Verordnungen zum Trotz gibt es keinerlei fairen Ausgleich zwischen Mensch und Tier und bei weitem keine Maximin-Lösung der Güterverteilung. Die Grundrechte der Tiere werden dem Gott des Geldes geopfert.

4) Schwieriger ist die Einordnung der Tierhaltung nach EU-Bio-Verordnung oder den strengeren Richtlinien der Ökolandwirtschafts-Verbände: Zweifelsohne ist sie deutlich besser als die Massentierhaltung. Aber darf sie schon als gerecht gelten? Es bleibt jedenfalls noch viel Luft nach oben, wenn man die Ausgaben der Menschen anderer Industrieländer für Lebensmittel mit den österreichischen oder deutschen vergleicht.

5) Relativ eindeutig gerecht zu sein scheint mir aber eine konsequent artgerechte und nachhaltige (und wo von den klimatischen Gegebenheiten her möglich, ganzjährige) Freilandhaltung. In diesem leider sehr seltenen Haltungssystem gibt der Bauer dem Tier eine sichere, vor Fressfeinden geschützte Weide mit ausreichend gutem Futter, eine stabile und geeignete soziale Gruppe, einen abwechslungsreichen Lebensraum, achtet auf die Bedürfnisse des Tieres in Gesundheit und Krankheit - bis zum Moment der Schlachtung auf der Weide oder dem Bauernhof, die schneller und sanfter abläuft als das langsame, oft qualvolle Verenden eines alten Wildtieres. Eine solche Haltungsform halte ich, wie leicht zu erkennen ist, für mindestens gleichwertig mit der „Haltungsform Gottes“, der freien Wildbahn. Die vernünftigen Vertragsparteien im Urzustand würden sie jederzeit wählen können.

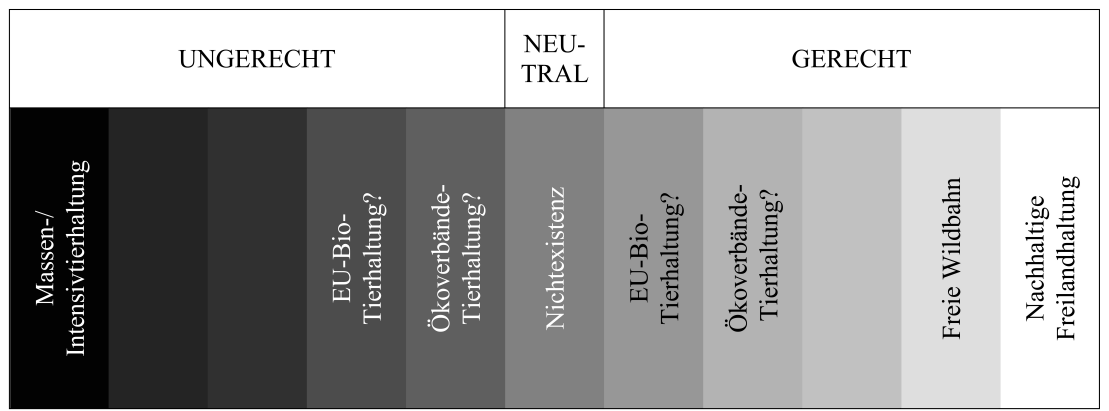


Über einzelne Aspekte meiner Einordnung mag man gerne diskutieren. Aber dass es Systeme der Tiernutzung einschließlich der Tiertötung geben kann, die ähnlich "gerecht" sind wie die freie Wildbahn und die die Ressourcen der Erde halbwegs fair zwischen menschlichen und nichtmenschlichen Tieren verteilen, scheint mir nicht zu bezweifeln. Freilich: Das überwältigende Gros der Tierhaltung in den Industrieländern erfüllt diese Gerechtigkeitsstandards nicht. Das liegt an den Lobbygruppen der „ProduzentInnen" ebenso wie an der Politik. Es liegt aber auch an den VerbraucherInnen, die möglichst billige Lebensmittel kaufen wollen. Den Mehrpreis einer Tierhaltung nach EU-Bio-Verordnung schätzt man auf zehn bis fünfzehn Prozent, nach den Richtlinien der Ökolandwirtschaftsverbände auf 20 bis 30 Prozent und einer konsequenten nachhaltigen Freilandhaltung auf etwa 100 Prozent. Wenn jemand wie eingangs vorgeschlagen nur noch ein Viertel der tierlichen Produkte konsumiert, bleiben in der Geldbörse unterm Strich also selbst in der teuersten Variante 50 Prozent des Geldes für tierliche Lebensmittel übrig. Und zugleich verliert die Gretchenfrage der Tierethik „Wie hältst du’s mit dem Töten?“ einen Großteil ihrer Schärfe.

\section{Literatur}

Bachl, Gottfried 2008, eucharistie. macht und lust des verzehrens, St. Ottilien.

Bäuerlein, Theresa 2011, Fleisch essen, Tiere lieben. Wo Vegetarier sich irren und was Fleischesser besser machen können, München.

Food and Agriculture Organization 2018, The State of World Fisheries and Aquaculture 2018 - Meeting the sustainable development goals, Rom.

Habermas, Jürgen 2001, Die Zukunft der menschlichen Natur, Frankfurt a. M.

Idel, Anita 2014, Die Kuh ist kein Klima-Killer. Zur Co-Evolution von Gras und Grasern und ihrer Bedeutung für die Potenziale nachhaltiger Land- und Weidewirtschaft, in: Voget-Kleschin, Lieske/Bossert, Leonie/Ott, Konrad (Hg.) 2014, Nachhaltige Lebensstile. Welchen Beitrag kann ein bewusster Fleischkonsum zu mehr Naturschutz, Klimaschutz und Gesundheit leisten?, Weimar/Lahn, 151-160.

Lintner, Martin M. 2017, Der Mensch und das liebe Vieh. Ethische Fragen im Umgang mit Tieren. Mit Beiträgen von Christoph J. Amor und Markus Moling, Innsbruck.

Loewe, Daniel 2008, Inclusión De Animales No-humanos En Un Marco De Argumentación Contractual, in: Veritas 53, 145-162.

Luick, Rainer 2014, Fleischgenuss und Landschaftsgestaltung, in: Voget-Kleschin, Lieske/Bossert, Leonie/Ott, Konrad (Hg.) 2014, Nachhaltige Lebensstile. Welchen Beitrag kann ein bewusster Fleischkonsum zu mehr Naturschutz, Klimaschutz und Gesundheit leisten?, Weimar/Lahn, 134-150. 
Nussbaum, Martha 2010, Die Grenzen der Gerechtigkeit. Behinderung, Nationalität und Spezieszugehörigkeit, Frankfurt a. M.

Peters, Anne 2019, Die Rechtsstellung von Tieren. Status quo und Weiterentwicklung, in: Diehl, Elke/Tuider, Jens (Hg.) 2019, Haben Tiere Rechte? Aspekte und Dimensionen der Mensch-Tier-Beziehung, Bonn, 122-134.

Rawls, John 1955, Two Concepts of Rules, in: The Philosophical Review 64, 3-32 (deutsch in: Höffe, Otfried [Hg.] 21992, Einführung in die utilitaristische Ethik, Tübingen, 135-166).

Rawls, John 1975, Eine Theorie der Gerechtigkeit, Frankfurt a. M.

Röhrs, Manfred/Sambraus, Hans Hinrich 1998, Tierhaltung, in: Lexikon der Bioethik 3, 539-554.

Rosenberger, Michael 2008, „Waid-Gerechtigkeit“. Grundzüge einer christlichen Ethik der Jagd, in: Lehr- und Forschungsanstalt für Land- und Forstwirtschaft (Hg.) 2008, Jagd und Jäger im Visier - Perspektiven für die Freizeitjagd in unserer Gesellschaft, Irdning, 5-14.

Rosenberger, Michael 2015, Der Traum vom Frieden zwischen Mensch und Tier. Eine christliche Tierethik, München.

Rosenberger, Michael 2016, Wie viel Tier darf's sein? Die Frage ethisch korrekter Ernährung aus christlicher Sicht, Würzburg.

Rosenberger, Michael 2018, Über individualethische Denkformen hinaus. (Tier-)Gerechtigkeit im Spannungsfeld individualistischer und systemischer Begründungen, in: Tierstudien 13, 124-135.

Rosenberger, Michael 2019, Allem Wild dienen. Überlegungen zu einer aus tierethischer Sicht vertretbaren Jagd, in: Diehl, Elke/Tuider, Jens (Hg.) 2019, Haben Tiere Rechte? Aspekte und Dimensionen der Mensch-Tier-Beziehung, Bonn, 271-278.

Rowlands, Mark 2002, Animals Like Us, London.

Rowlands, Mark 22009, Animal Rights. Moral Theory and Practice, New York.

Ruhstorfer, Karlheinz 2012, Systematische Theologie Modul 3, Paderborn.

Schmitz, Friederike 2015, Vertragstheorie, in: Petrus, Klaus/Ferrari, Arianna (Hg.) 2015, Lexikon der Mensch/Tier-Beziehungen, Bielefeld, 413-415.

Stucki, Saskia 2016, Grundrechte für Tiere. Eine Kritik des geltenden Tierschutzrechts und rechtstheoretische Grundlegung von Tierrechten im Rahmen einer Neupositionierung des Tieres als Rechtssubjekt, Baden-Baden.

United States Department of Agriculture (USDA) 2011, Putting Dairy Cows Out to Pasture. An Environmental Plus, in: Agricultural Research magazine May/June 2011, http://agresearchmag.ars.usda.gov/2011/may/cows (abgerufen am 15.10.2019).

Wildt, Andreas 1998, Menschenrechte und moralische Rechte, in: Gosepath, Stefan/Lohmann, Georg (Hg.) 1998, Philosophie der Menschenrechte, Frankfurt a. M., 124-145.

Zsifkovits, Valentin 2012, Orientierungen für eine humane Welt, Wien/Berlin. 